\title{
Neoadjuvant and adjuvant chemotherapy with doxorubicin and ifosfamide for bone sarcomas in adult and older patients
}

\author{
HIROSHI URAKAWA ${ }^{1}$, SATOSHI TSUKUSHI ${ }^{1}$, HIDESHI SUGIURA ${ }^{2}$, KENJI YAMADA ${ }^{3}$, YOSHIHISA YAMADA ${ }^{4}$, \\ EIJI KOZAWA ${ }^{1}$, EISUKE ARAI ${ }^{1}$, NAOHISA FUTAMURA ${ }^{1}$, NAOKI ISHIGURO $^{1}$ and YOSHIHIRO NISHIDA $^{1}$ \\ ${ }^{1}$ Department of Orthopaedic Surgery, Nagoya University Graduate School and School of Medicine, Nagoya, Aichi 466-8550; \\ ${ }^{2}$ Department of Orthopedic Surgery, Aichi Cancer Center Hospital, Nagoya, Aichi 464-8681; \\ ${ }^{3}$ Orthopedic Surgery, Aichi Cancer Center Aichi Hospital, Okazaki, Aichi 444-0011; \\ ${ }^{4}$ Orthopedic Surgery, Nagoya Memorial Hospital, Nagoya, Aichi 468-8520, Japan
}

Received February 12, 2014; Accepted September 8, 2014

DOI: $10.3892 / 01.2014 .2567$

\begin{abstract}
The present study investigated the safety and efficacy of neoadjuvant and adjuvant chemotherapy with doxorubicin and ifosfamide for bone sarcoma in adult and older patients. A total of 18 consecutive patients with bone sarcoma (American Joint Committee on Cancer stage II in 14 patients and stage IV in four) treated with neoadjuvant and adjuvant chemotherapy at Nagoya Musculoskeletal Oncology Group hospitals in Japan between 2004 and 2011 were reviewed. The treatment efficacy and side-effects were evaluated. The responses to neoadjuvant chemotherapy were stable disease in 11 patients and progressive disease in three. Among the 12 evaluable patients, there were five with $\geq 90 \%$ tumor necrosis. The estimated overall survival (OS) rate at five years for the patients without metastasis prior to treatment was $56 \%$. Major grade 3 or 4 side-effects included leukopenia in 14 cases, anemia in seven, thrombocytopenia in three, nausea in two and febrile neutropenia in two. One patient discontinued chemotherapy due to a temporarily depressed level of consciousness with arrhythmia (grade 2). The estimated five-year OS rate in this study was acceptable in patients without metastasis prior to treatment. A better coordinated prospective study of this combination regimen for older patients with bone sarcoma will be required to clarify its efficacy and tolerability.
\end{abstract}

\section{Introduction}

There are two peaks of osteosarcoma incidence, as determined by population-based surveillance (1). The first is during adolescence, and the later peak is in the eighth decade. Neoadjuvant and adjuvant chemotherapy protocols have improved long-term survival, particularly in young patients with osteosarcoma. In

Correspondence to: Dr Hiroshi Urakawa, Department of Orthopaedic Surgery, Nagoya University Graduate School and School of Medicine, 65 Tsurumai, Showa-ku, Nagoya, Aichi 466-8550, Japan

E-mail: urakawa@med.nagoya-u.ac.jp

Key words: doxorubicin, ifosfamide, chemotherapy, bone sarcoma population-based data, the relative five-year survival rates are 61.6, 58.7, and 24.2\% in osteosarcoma patients aged 0-24, 25-59 and 60-85 years, respectively (1), with older patients having a poorer prognosis. Malignant fibrous histiocytoma (MFH) and dedifferentiated chondrosarcoma are known to occur in elderly patients $(2,3)$.

Phase II studies of single agents and combinations of drugs have documented the efficacy of methotrexate (MTX), doxorubicin (DXR) and cisplatin (CDDP) in advanced osteosarcoma (4-6), leading to randomized studies confirming the efficacy of adjuvant chemotherapy $(7,8)$. This three-drug combination represents the standard care for osteosarcoma. Ifosfamide (IFM), in studies with other agents, has been shown to exhibit significant activity in recurrent or metastatic osteosarcoma $(9,10)$. However, IFM did not improve the degree of tumor necrosis in a study of patients with osteosarcoma who were treated with a neoadjuvant MTX, DXR, and CDDP regimen, with or without IFM (11).

Neoadjuvant and adjuvant chemotherapy protocols have not been established specifically for adult and older patients with bone sarcomas. Furthermore, the completion rate of regimens that have included CDDP and/or MTX has been reported to be low in this population $(12,13)$. High completion rates of neoadjuvant and adjuvant chemotherapy with DXR and IFM have been reported in older patients with soft-tissue sarcoma $(14,15)$. The present study was undertaken to determine the safety and efficacy of neoadjuvant and adjuvant chemotherapy with DXR and IFM for bone sarcoma in adult and older patients.

\section{Patients and methods}

Patients. Patients with bone sarcoma treated with neoadjuvant and adjuvant chemotherapy in four Nagoya Musculoskeletal Oncology Group Hospitals (Nagoya University Hospital, Aichi Cancer Center Hospital, Aichi Cancer Center Aichi Hospital and Nagoya Memorial Hospital) in Japan between January 2004 and February 2011 were reviewed. Patients aged $>40$ years with osteosarcoma and $\mathrm{MFH}$ of the bone were prospectively treated with IFM+DXR in these institutions. Other bone sarcomas in patients of all ages were treated with IFM+DXR according to the attending physician's decision. 
After obtaining a waiver of patient informed consent requirements from the institutional review board, 18 consecutive patients with bone sarcoma were retrospectively reviewed. This study was approved by the ethics committee of Nagoya University Graduate School and School of Medicine (Nagoya, Japan).

Patient characteristics and treatment regimens. Baseline demographic and clinical characteristics are listed in Table I. The study group consisted of 12 males and six females, with a median age of 63 years (range, 28-76 years). Histological subtypes were osteosarcoma $(n=10), M F H$ of the bone $(n=4)$, dedifferentiated chondrosarcoma $(n=3)$ and angiosarcoma of the bone $(n=1)$. According to the staging system of the American Joint Committee on Cancer (16), there were four patients of stage IIA, ten of stage IIB, one of stage IVA and three of stage IVB. The timing of chemotherapy was neoadjuvant and adjuvant in seven patients, neoadjuvant only in seven and adjuvant only in four. The median number of cycles of chemotherapy was 4 (range, 1-7). Patients were treated with neoadjuvant and/or adjuvant chemotherapy with $50-60 \mathrm{mg} / \mathrm{m}^{2}$ DXR and 6-10 g/m $/ \mathrm{m}^{2}$ IFM every 3-4 weeks.

Treatment analysis. Side-effects were graded according to the Common Terminology Criteria for Adverse Events v4.0 (17). The treatment efficacies were evaluated according to Response Evaluation Criteria In Solid Tumors (RECIST) v1.1 analysis of magnetic resonance imaging (18), necrosis rate of resected tumor and patient outcome.

Statistical analysis. The overall survival (OS) rate from definitive treatment was calculated using Kaplan-Meier product limit methods. A log-rank test was used to identify differences in survival rates between groups. $\mathrm{P}<0.05$ was used to indicate a statistically significant difference.

\section{Results}

Definitive treatments. One patient with metastasis could not undergo definitive surgery due to disease progression during neoadjuvant chemotherapy. The sites of definitive treatment for the primary tumors were the femur $(n=4)$, tibia $(n=3)$, humerus $(n=2)$, pelvis $(n=2)$, sternum $(n=2)$ and spine $(n=1)$ in patients without metastasis. The metastatic sites of definitive treatment for patients with metastatic tumors were the lung only $(n=1)$, the lymph nodes only $(\mathrm{n}=1)$, and the bone and soft tissues $(\mathrm{n}=1)$. Definitive treatments were surgery in 15 cases, heavy ion radiation in one case and surgery and heavy ion radiation in one case each. Sites of definitive treatment were primary of the extremity in nine cases, primary of the trunk in five, primary and metastasis in two, and solitary distant recurrence in one.

Treatment response. Evaluation with RECIST for the treatment site showed that the responses following neoadjuvant chemotherapy, with a median number cycles of 3.5 (range, 1-5), were stable disease (SD) in 11 cases (79\%) and progressive disease (PD) in three (21\%) (Table II). Of the 12 patients with $\mathrm{SD}$ and PD, there were five $(42 \%)$ with tumor necrosis of $\geq 90 \%$ (Table II). The final status was no evidence of disease in nine cases (50\%), alive without disease in two (11\%) and
Table I. Baseline demographics and clinical characteristics

\begin{tabular}{|c|c|}
\hline Characteristic & Value \\
\hline \multicolumn{2}{|l|}{ Gender, n (\%) } \\
\hline Male & $12(66)$ \\
\hline Female & $6(34)$ \\
\hline \multicolumn{2}{|l|}{ Histological subtype, n (\%) } \\
\hline Osteosarcoma & $10(56)$ \\
\hline $\mathrm{MFH}$ of the bone & $4(22)$ \\
\hline Dedifferentiated chondrosarcoma & $3(17)$ \\
\hline Angiosarcoma of the bone & $1(6)$ \\
\hline \multicolumn{2}{|l|}{ AJCC stage, n $(\%)$} \\
\hline IIA & $4(22)$ \\
\hline IIB & $10(56)$ \\
\hline IVA & $1(6)$ \\
\hline IVB & $3(17)$ \\
\hline \multicolumn{2}{|l|}{ Definitive treatment, $\mathrm{n}(\%)$} \\
\hline Surgery & $15(83)$ \\
\hline Heavy ion radiation & $1(6)$ \\
\hline Surgery and heavy ion radiation & $1(6)$ \\
\hline Inoperable with PD & $1(6)$ \\
\hline \multicolumn{2}{|c|}{ Site of definitive treatment $(n=17), n(\%)$} \\
\hline Primary, extremity & $9(53)$ \\
\hline Primary, trunk & $5(29)$ \\
\hline Primary and metastasis & $2(12)$ \\
\hline Solitary distant recurrence & $1(6)$ \\
\hline \multicolumn{2}{|l|}{ Timing of chemotherapy, n (\%) } \\
\hline Neoadjuvant only & $7(39)$ \\
\hline Adjuvant only & $4(22)$ \\
\hline Neoadjuvant and adjuvant & $7(39)$ \\
\hline Median age, years (range) & $63(28-76)$ \\
\hline Median number of & $4(1-7)$ \\
\hline
\end{tabular}

chemotherapy cycles (range)

Median follow up, months (range)

$18.0(4.6-73.7)$

MFH, malignant fibrous histiocytoma; AJCC, American Joint Committee on Cancer; PD, progressive disease.

dead of disease in seven (39\%), at a median follow-up time of 18.0 months (range, 4.6-73.7 months) (Table II). Estimated five-year OS rates for patients without and with metastasis prior to treatment were $56 \%$ (Fig. 1) and 25\% (Fig. 2), respectively (Table II). Estimated two-year OS rates for patients without metastasis were $100 \%$ in patients aged $<60$ years, and $54 \%$ in patients $>60$ years ( $\mathrm{P}=0.072$ between the two groups) (Fig. 3).

One patient discontinued chemotherapy due to a temporarily depressed level of consciousness with arrhythmia (grade 2). Major grade 3 or 4 side-effects included leukopenia in 14 cases $(78 \%)$, anemia in seven (39\%), thrombocytopenia in three (17\%), nausea in two (11\%), and febrile neutropenia in two (11\%). Cardiotoxicity was not observed in the follow-up periods. 
Table II. Efficacy data.

\begin{tabular}{lr}
\hline Response category & Value \\
\hline RECIST response $(\mathrm{n}=14), \mathrm{n}(\%)$ & $11(79)$ \\
SD & $3(21)$ \\
PD & \\
Tissue response in SD and PD $(\mathrm{n}=12), \mathrm{n}(\%)$ & $5(42)$ \\
Necrosis $\geq 90 \%$ & $7(58)$ \\
Necrosis $<90 \%$ & \\
Final status (n=18), $\mathrm{n}(\%)$ & $9(50)$ \\
NED & $2(11)$ \\
AWD & $7(39)$ \\
DOD & \\
Estimated five-year OS rate, $\%$ & 56 \\
AJCC stage II & 25 \\
AJCC stage IV & \\
\hline
\end{tabular}

RECIST, response evaluation criteria in solid tumors; SD, stable disease; $\mathrm{PD}$, progressive disease; NED, no evidence of disease; AWD, alive with disease; DOD, dead of disease; OS, overall survival, AJCC, American Joint Committee on Cancer.

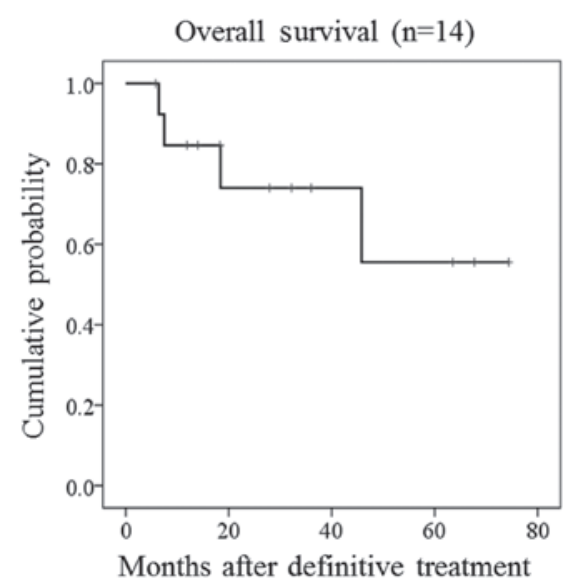

Figure 1. Kaplan-Meier estimated overall survival for 14 osteosarcoma patients without metastasis.

\section{Discussion}

The number of aged osteosarcoma patients is increasing $(19,20)$. Chemotherapy with DXR, CDDP and high-dose MTX has become a standard neoadjuvant and adjuvant treatment for osteosarcoma of young patients. However, older patients have been reported to receive less chemotherapy and have a poorer outcome (19). Since the completion rate of regimens, including CDDP and/or MTX, has been reported to be low in middle-aged and older bone sarcoma patients $(12,13)$, a chemotherapeutic regimen with DXR and IFM was prospectively applied to adult and older patients with bone sarcomas in the present study. This regimen has already been widely used for adult patients with soft-tissue sarcoma $(15,21)$. In the present study, only one patient discontinued chemotherapy due to a temporarily depressed level of consciousness with

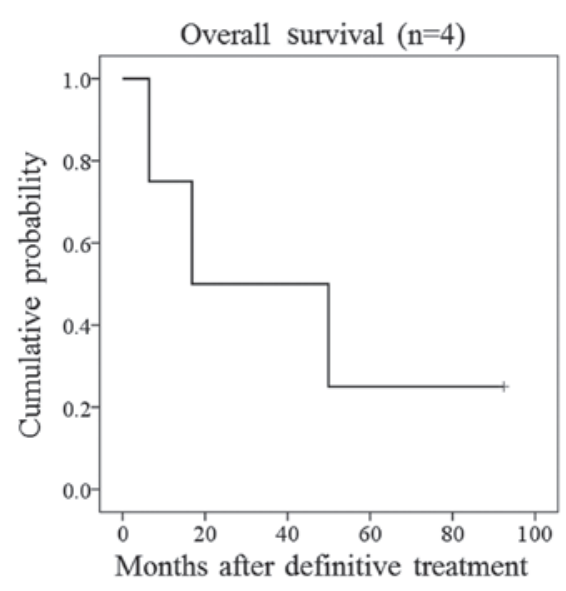

Figure 2. Kaplan-Meier estimated overall survival for four osteosarcoma patients with metastasis.

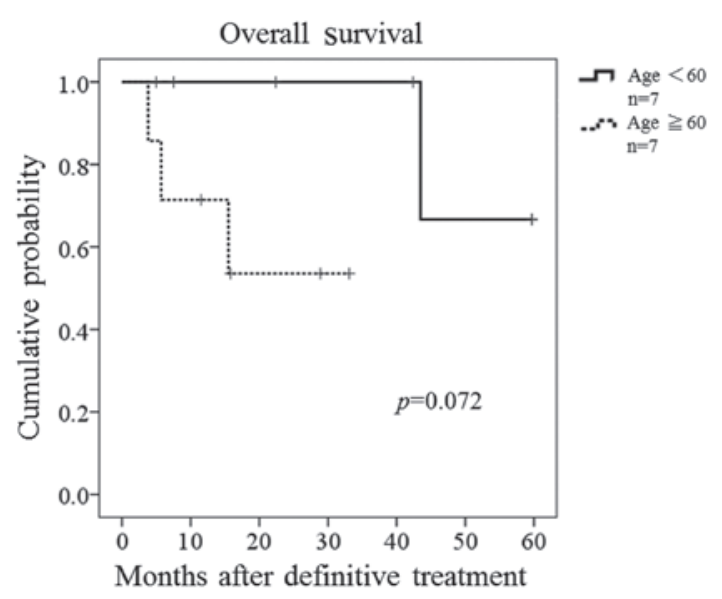

Figure 3. Kaplan-Meier estimated overall survival for seven osteosarcoma patients aged $<60$ years and seven patients aged $>60$ years without metastasis. There was no statistical difference between the two groups $(\mathrm{P}=0.072)$.

arrhythmia, suggesting that the combination of DXR and IFM is well tolerated, even in patients $>60$ years (22).

High-dose IFM treatment was reported to be associated with a higher incidence of azoospermia (23). However, this is less of a disadvantage with respect to infertility in the case of older patients. DXR-induced cardiotoxicity has been reported in the long-term follow-up of osteosarcoma (24); however no cardiotoxicity was noted in the present study, albeit within only a short median follow-up period of 18.0 months (range, 4.6-73.7 months).

Bramwell et al reported that adjuvant and/or neoadjuvant chemotherapy is beneficial in patients with MFH of the bone (13). The merit of adjuvant and/or neoadjuvant chemotherapy for dedifferentiated chondrosarcoma has not been proven (3). However, the present study performed adjuvant chemotherapy with IFM and DXR due to the poor outcome in patients with the sarcoma.

Previous studies have demonstrated $\geq 90 \%$ tumor necrosis in $21-48 \%$ of cases following neoadjuvant chemotherapy in osteosarcoma patients aged $>40$ years $(12,19,25,26)$. In a study on MFH of the bone, $\geq 90 \%$ tumor necrosis was observed in $42 \%$ of patients subsequent to chemotherapy (13). In the present study, the responses following neoadjuvant chemotherapy 
were SD in 11 cases and PD in three. Five of 12 patients (42\%) with bone sarcoma had $\geq 90 \%$ necrosis, and the efficacy of this treatment was acceptable.

In studies on neoadjuvant and/or adjuvant chemotherapy for non-metastatic osteosarcoma of an extremity, the estimated five-year OS rate was reported as $55-70 \%$ in patients aged $40-60$ years $(12,25)$ and $51 \%$ in patients $>40$ years (19). In MFH of the bone, the five-year OS rate following neoadjuvant and/or adjuvant chemotherapy was 59\% in non-metastatic patients with a median age of 42 years (range, 14-62 years) (13). In non-metastatic patients aged $<60$ years who underwent limb salvage for dedifferentiated chondrosarcoma, the five-year OS rates were reported as $25 \%$ in those who did not have chemotherapy compared with $45 \%$ in those who did. However, no significant difference was noted between these groups on univariate analysis (3). In the present study, even though there were five tumors located in the axial skeleton and nine in the extremities, and the median age of the patients was 63 years (range, 28-76 years), the five-year OS rate of $56 \%$ was comparable to that of previous reports regarding bone sarcoma in patients without metastasis $(3,12,13,19,25)$.

There were certain limitations to the present study. The first was the diversity of the chemotherapy dose and cycles based on the attending physician's decision. A combination regimen with DXR $\left(60 \mathrm{mg} / \mathrm{m}^{2}\right)$ and IFM $\left(10 \mathrm{~g} / \mathrm{m}^{2}\right)$ was typically selected, used in four cycles prior to surgery and two cycles subsequent to surgery, but certain cases were treated with different doses and cycles according to the attending physician's decision. The second limitation was the short follow-up periods in this study. In particular, the follow-up periods in the older patients were short, and did not allow calculation of the estimated OS rate at five years in this population. Furthermore, the study was unable to evaluate cardiotoxicity over a long follow-up period. Finally, the study included various bone sarcomas, including osteosarcoma, cases of adult and older patients, and extremity and trunk sites. However, the results may be useful in demonstrating the clinical features of bone sarcoma of various subtypes, sites and age in actual practice.

In conclusion, the estimated five-year OS rate and necrosis rate following neoadjuvant and adjuvant chemotherapy with DXR and IFM for bone sarcomas in this study were acceptable in patients without metastasis prior to treatment. A better coordinated prospective study of this combination regimen for aged patients with bone sarcoma will be required to clarify its efficacy and tolerability.

\section{Acknowledgements}

Funding was granted from the Ministry of Education, Culture, Sports, Science and Technology of Japan (Grant-in-Aid for Scientific Research, no. 24791533). The authors would like to thank Miss. Eri Ishihara for providing secretarial assistance.

\section{References}

1. Mirabello L, Troisi RJ and Savage SA: Osteosarcoma incidence and survival rates from 1973 to 2004: data from the Surveillance, Epidemiology, and End Results Program. Cancer 115: 1531-1543, 2009.

2. Weiss SW and Enzinger FM: Malignant fibrous histiocytoma: an analysis of 200 cases. Cancer 41: 2250-2266, 1978.

3. Grimer RJ, Gosheger G, Taminiau A, et al: Dedifferentiated chondrosarcoma: prognostic factors and outcome from a European group. Eur J Cancer 43: 2060-2065, 2007.
4. Cores EP, Holland JF, Wang JJ and Sinks LF: Doxorubicin in disseminated osteosarcoma. JAMA 221: 1132-1138, 1972.

5. Jaffe N, Paed D, Farber S, et al: Favorable response of metastatic osteogenic sarcoma to pulse high-dose methotrexate with citrovorum rescue and radiation therapy. Cancer 31: 1367-1373, 1973.

6. Ochs JJ, Freeman AI, Douglass HO Jr, Higby DS, Mindell ER and Sinks LF: cis-Dichlorodiammineplatinum (II) in advanced osteogenic sarcoma. Cancer Treat Rep 62: 239-245, 1978.

7. Link MP, Goorin AM, Miser AW, et al: The effect of adjuvant chemotherapy on relapse-free survival in patients with osteosarcoma of the extremity. N Eng J Med 314: 1600-1606, 1986.

8. Eilber F, Giuliano A, Eckardt J, Patterson K, Moseley S and Goodnight J: Adjuvant chemotherapy for osteosarcoma: a randomized prospective trial. J Clin Oncol 5: 21-26, 1987.

9. Miser JS, Kinsella TJ, Triche TJ, et al: Ifosfamide with mesna uroprotection and etoposide: an effective regimen in the treatment of recurrent sarcomas and other tumors of children and young adults. J Clin Oncol 5: 1191-1198, 1987.

10. Goorin AM, Harris MB, Bernstein M, et al: Phase II/III trial of etoposide and high-dose ifosfamide in newly diagnosed metastatic osteosarcoma: a pediatric oncology group trial. J Clin Oncol 20: 426-433, 2002.

11. Ferrari S, Ruggieri P, Cefalo G, et al: Neoadjuvant chemotherapy with methotrexate, cisplatin, and doxorubicin with or without ifosfamide in nonmetastatic osteosarcoma of the extremity: an Italian sarcoma group trial ISG/OS-1. J Clin Oncol 30: 2112-2118, 2012.

12. Song WS, Kong CB, Jeon DG, et al: Prognosis of extremity osteosarcoma in patients aged 40-60 years: a cohort/case controlled study at a single institute. Eur J Surg Oncol 36: 483-488, 2010.

13. Bramwell VH, Steward WP, Nooij M, et al: Neoadjuvant chemotherapy with doxorubicin and cisplatin in malignant fibrous histiocytoma of bone: A European Osteosarcoma Intergroup study. J Clin Oncol 17: 3260-3269, 1999.

14. Woll PJ, Reichardt P, Le Cesne A, et al; EORTC Soft Tissue and Bone Sarcoma Group and the NCIC Clinical Trials Group Sarcoma Disease Site Committee: Adjuvant chemotherapy with doxorubicin, ifosfamide, and lenograstim for resected soft-tissue sarcoma (EORTC 62931): a multicentre randomised controlled trial. Lancet Oncol 13: 1045-1054, 2012.

15. Frustaci S, Gherlinzoni F, De Paoli A, et al: Adjuvant chemotherapy for adult soft tissue sarcomas of the extremities and girdles: results of the Italian randomized cooperative trial. J Clin Oncol 19: 1238-1247, 2001.

16. Edge SB, Byrd DR, Compton CC, et al (eds): Bone. In: AJCC Cancer Staging Manual. 7th edition. New York, Springer, New York, NY, pp281-290, 2010.

17. National Cancer Institute: National Cancer Institute Common Toxicity Criteria version 4.0. http://evs.nci.nih.gov/ftp1/ CTCAE/. Accessed September 10, 2013.

18. Eisenhauer EA, Therasse P, Bogaerts J, et al: New response evaluation criteria in solid tumours: revised RECIST guideline (version 1.1). Eur J Cancer 45: 228-247, 2009.

19. Grimer RJ, Cannon SR, Taminiau AM, et al: Osteosarcoma over the age of forty. Eur J Cancer 39: 157-163, 2003.

20. Nishida Y, Isu K, Ueda T, et al: Osteosarcoma in the elderly over 60 years: a multicenter study by the Japanese Musculoskeletal Oncology Group. J Sur Oncol 100: 48-54, 2009.

21. Comandone A, Bretti S, Bertetto O, Oliva C, Bergnolo P and Bumma C: Low dose adriamycin and ifosfamide in the treatment of advanced adult soft tissue sarcomas. Anticancer Res 20: 2077-2080, 2000.

22. Urakawa H, Tsukushi S, Tsurudome I, et al: Metastasis of osteosarcoma to stomach made clinically evident by hematemesis: a case report. World J Surg Oncol 11: 48, 2013.

23. Longhi A, Macchiagodena M, Vitali G and Bacci G: Fertility in male patients treated with neoadjuvant chemotherapy for osteosarcoma. J Pediatr Hematol/Oncol 25: 292-296, 2003.

24. Bacci G, Ferrari S, Bertoni F, et al: Long-term outcome for patients with nonmetastatic osteosarcoma of the extremity treated at the istituto ortopedico rizzoli according to the istituto ortopedico rizzoli/osteosarcoma-2 protocol: an updated report. J Clin Oncol 18: 4016-4027, 2000.

25. Bacci G, Ferrari S, Mercuri M, et al: Neoadjuvant chemotherapy for osteosarcoma of the extremities in patients aged 41-60 years: outcome in 34 cases treated with adriamycin, cisplatinum and ifosfamide between 1984 and 1999. Acta Orthop 78: 377-384, 2007.

26. Manoso MW, Healey JH, Boland PJ, et al: De novo osteogenic sarcoma in patients older than forty: benefit of multimodality therapy. Clin Orthop Relat Res 438: 110-115, 2005. 